\title{
Radiation recall masquerading as an infectious process
}

\begin{abstract}
Cellulitis and other infections are common in cancer patients, especially patients who are immunosuppressed following chemotherapy. When patients do not respond to antimicrobial therapy as expected, it is imperative for clinicians to consider other treatment-related conditions in the differential diagnosis. One such condition unique to cancer patients who have received radiation therapy is radiation recall, which may masquerade as an infectious phenomenon and can be a recurrent issue in a previously irradiated area. We report a case of radiation recall cellulitis and myositis during treatment with gemcitabine, cisplatin, and nab-paclitaxel after radiation therapy. Upfront identification of radiation recall facilitates its timely diagnosis and management.
\end{abstract}

Keywords: radiation, cellulitis, myositis, infection
Volume 2 Issue 6 - 2017

\author{
Santhosshi Narayanan,' Bilal Mujtaba, ${ }^{2}$ John \\ Eugene Koay, ${ }^{3}$ Mohamed Elshikh, ${ }^{2}$ John E \\ Madewell, ${ }^{2}$ Gauri R Varadhachary ${ }^{4}$ \\ 'Department of Internal Medicine, University of TX MD \\ Anderson Cancer Center, USA \\ ${ }^{2}$ Department of Diagnostic Imaging, University of TX MD \\ Anderson Cancer Center, USA \\ ${ }^{3}$ Department of Radiation Oncology, University of TX MD \\ Anderson Cancer Center, USA \\ ${ }^{4}$ Department of Medical Oncology, University of TX MD \\ Anderson Cancer Center, USA
}

Correspondence: Bilal Mujtaba, Department of Diagnostic Imaging, University of TX MD Anderson Cancer Center, USA, Tel 2034350947,Email bmujtaba@mdnaderson.org

Received: April 27, 2017 | Published: May 03, 2017

\section{Introduction}

Patients with cancer often receive treatment with multiple modalities including radiation, chemotherapy, surgery, and immunotherapy. Patients treated with radiation can be sensitized to and can develop a delayed inflammatory response to chemotherapy agents or other drugs in the irradiated site. This phenomenon is called radiation recall and can involve the skin, soft tissues or various organs depending on the site of radiation therapy. Dermatitis, duodenitis, colitis, optic neuritis, pneumonitis, gastritis based radiation recall conditions have been described in the literature.

Here-in, we describe a case of radiation recall that was initially diagnosed as infectious cellulitis, progressed to severe myositis despite treatment with antibiotics, and eventually responded well to steroids. The presentation was particularly challenging since patient had an intramedullary nail in place for treatment of pathologic fracture. Although radiation recall is a known phenomenon, its presentation as myositis is uncommon.

\section{Case presentation}

A 44year-old woman presented to the gastrointestinal medical oncology clinic with pain and swelling of her left arm. She was being treated at our institution for metastatic cholangiocarcinoma which was diagnosed after she presented with a pathological fracture of her humerus (Figure 1A \& 1B).The fracture was treated with intramedullary nailing 4 months prior to her presentation. After surgery, she underwent radiation therapy to the left arm; 30Gy in 10 fractions using 2 opposed beams covering the metastasis and surgical hardware (Figure 1A \& 1B). After completing radiation therapy, she was enrolled in a front line clinical trial with gemcitabine, cisplatin and nab-paclitaxel; she had received 5 cycles at the time of presentation with arm swelling.

During the initial work up, venous ultrasonography was negative for deep vein thrombosis. Magnetic resonance imaging (MRI) of the left upper extremity showed no evidence of abscess (Figure 2A). No recurrence of metastasis was seen. She was afebrile with normal laboratory studies and was referred to the institution's lymphedema clinic and proceeded to receive her next cycle of chemotherapy.

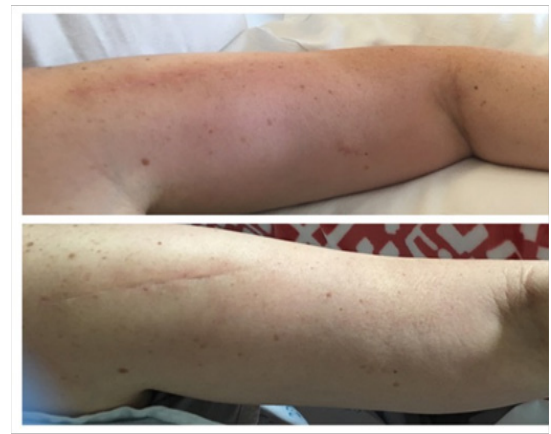

Figure I (A) Baseline presentation of erythema and edema. (B) 48hours after treatment with steroids.

Two months after the initial presentation she was hospitalized for severe pain in the area of the intramedullary nail and worsening swelling of her left arm and fever (Tmax 100.3 degrees Fahrenheit). Laboratory studies revealed leukocytosis, with a white blood cell count of $29000 / \mu \mathrm{L}$. Plain radiographs taken at this time were unremarkable. MRI scans showed diffuse soft-tissue edema in the left upper arm. Given the concern of an infected prosthesis, after consultation with orthopedic surgery and infectious disease teams, blood cultures were obtained, and broad spectrum antibiotics were administered to empirically treat MRSA (Methicillin resistant staphylococcus aureus) and gram-negative infections. One of the two blood cultures performed was positive for Staphylococcus hominis. The patient was monitored and had minimal response to antibiotics with a mild reduction in swelling. S. hominis was thought to be likely a contaminant and unlikely to cause a bone or soft-tissue infection, but because of the bacteremia and the low-grade fever, the decision was made to treat 
the condition as infectious cellulitis. Treatment with daptomycin and cefepime was begun; her initial creatine phosphokinase level (CPK) was 40U/L. She was discharged on intravenous Daptomycin and Cefepime as outpatient.

One week after discharge, she developed increasing pain and was readmitted to the hospital. Physical examination of her left arm revealed severe edema with erythema. Repeat blood cultures were obtained, which were negative for $S$. hominis or other organisms. Her CPK level during this admission was 372U/L. Skin and muscle biopsy were considered but not pursued due to the risk of impending compartment syndrome. Repeat MRI scans showed progressive cellulitis and myositis (Figure 2B). Inflammatory/autoimmune etiologies were considered in the differential diagnosis. Radiation recall was suspected because her myositis pattern was localized to the site of prior radiation therapy and her symptoms were progressive despite the use of broad-spectrum antibiotics. Additionally, there is a known relationship between use of these chemotherapies and radiation recall.

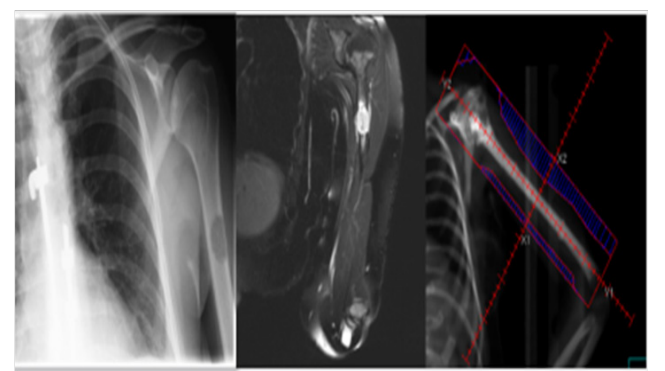

Figure 2 Preoperative radiography (Panel A) and magnetic resonance imaging (Panel B) show a lytic lesion on the humerus (blue arrows). A radiation treatment plan shows the target area (Panel C).

Empiric therapy with steroids was begun immediately, which resulted in a dramatic reduction in her arm swelling and pain (Figure $3 \mathrm{~A} \& 3 \mathrm{~B}$ ). She was discharged in 4 days in a stable condition. On follow-up, her symptoms had completely resolved. Despite presence of stable disease on her last imaging, the decision was made for her to discontinue protocol treatment with gemcitabine, cisplatin and nabpaclitaxel because these drugs are individually known to be associated with radiation recall response (gemcitabine more than the other two).

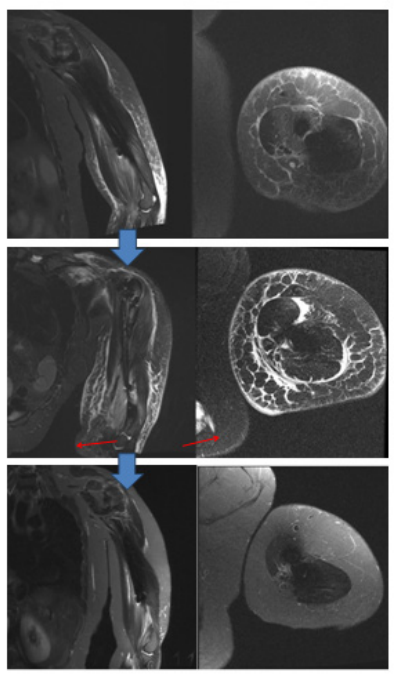

Figure 3 Baseline postoperative MRI, coronal and sagittal planes, show no evidence of abscess (Panel A). MRI scans on readmission show cellulitis and myositis characterized by increasing edema (red arrows) in soft tissues and muscle (Panel B). Panel C MRI showing resolution of edema on follow up visit after discharge from the hospital.

\section{Discussion}

The phenomenon in which chemotherapy drugs reactivate latent radiation effects in previously irradiated normal tissues was first reported in 1959 in children. ${ }^{1}$ The true incidence of this condition unclear due to the limited literature, but observational studies mostly involving cutaneous reactions indicates a wide range varying between $1.8 \%$ and $11.5 \% .^{2-6}$

The mechanism of radiation recall response is unknown. One theory suggests drug hypersensitivity and local vasculopathy in the irradiated area as inducing factors. ${ }^{7}$ Radiation therapy causes depletion of stem cells in the treatment field, thereby predisposing the tissue to future insults from drugs. ${ }^{8}$ In contrast to radiosensitization, which occurs within 7 days of radiation exposure, radiation recall reactions can happen weeks or evenyears after radiation therapy, when chemotherapy or another offending agent is introduced. The time interval between the completion of radiation therapy and the initiation of chemotherapy that triggers the radiation recall is usually 5 months or less, but as high as 25 years' lapse have been reported in the literature. ${ }^{9}$

Radiation recall usually manifests as a cutaneous reaction, although inflammation of the bowel, ${ }^{10}$ lungs, ${ }^{11}$ supraglottic, ${ }^{12}$ central nervous syste, ${ }^{13}$ and muscles can occur. Many chemotherapeutic agents such as 5-fluorouracil, capecitabine, taxanes (eg, docetaxel and paclitaxel), anthracyclines (eg, doxorubicin), oxaliplatin, cisplatin, carboplatin, and molecular targeted agents (eg, sunitinib) can precipitate these reactions; in addition, cases have been reported in which radiation recall was triggered by antibiotics (eg, gatifloxacin), codeine, simvastatin, levetiracetam, and other drugs. Our patient received gemcitabine, nab-paclitaxel, and cisplatin, all of which have been reported to cause radiation recall mysotitis. ${ }^{14}$ Gemcitabine seems to disproportionally affect internal tissues and organs. In a review of 13 patients with gemcitabine-associated radiation recall, only 4 patients presented with dermatitis or mucositis while 4 presented with myositis. $^{15}$

The use of immunotherapy drugs, especially programmed cell death protein 1 (PD-1) inhibitors, is increasing, and there is a risk of immune-related adverse events with their use. Radiation produces neoantigens in the tissues, ${ }^{16}$ and PD-1 inhibitors' enhancement of the immune system to attack the tumor antigens could theoretically lead to an increase in inflammation of tissues exposed to radiation. More laboratory and clinical studies are needed to understand the role of neoantigens in the inflammatory cascade and radiation recall. ${ }^{17}$

Radiation recall cellulitis and myositis manifests as erythema, warmth, and pain, making radiation recall indistinguishable from infectious cellulitis; most such cases are diagnosed when the disease does not respond to the initial antibiotic regimen. Other conditions to consider in the differential diagnosis are inflammatory and autoimmune myopathies. Radiation recall reactions are confined to sites of previous irradiation, which is a clue to accurate diagnosis. CPK may be elevated in cases of radiation recall myositis, and MRI is helpful to exclude abscess or hemorrhage-especially in patients with thrombocytopenia from recent chemotherapy. Failure to recognize and treat radiation recall causes continued physical distress and can lead to serious effects such as compartment syndrome. ${ }^{18}$ A skin biopsy may help rule out other etiologies; however, negative microbial cultures from the biopsy may not rule out infectious etiology, as the yield is low in skin biopsies.

The management of radiation recall reactions includes the withdrawal of offending agents and administration of steroids. 
One case report described the continuation of gemcitabine for 7 months with concurrent use of oral steroids. ${ }^{19}$ Discontinuation of gemcitabine alone resulted in complete resolution in few cases, ${ }^{20}$ and there are reports of unsuccessful re-challenge with gemcitabine. Thus, the decision whether to continue or withdraw offending agents remains controversial. ${ }^{21}$ Although steroids are commonly used in the management of radiation recall, the use of ibuprofen with vitamins $\mathrm{C}$ and $\mathrm{E}$ was in 1 case report. ${ }^{22}$ Immediate improvement has been reported with systemic steroids, which were followed by a short tapering regimen. However, some patients may require a prolonged steroid tapering regimen. ${ }^{23}$

\section{Conclusion}

it is important for clinicians to be aware of radiation recall response reactions and recognize symptoms immediately to allow appropriate management and prevent morbidity for affected patients.

\section{Acknowledgements}

None.

\section{Conflict of interest}

Author declares that there is no conflict of interest.

\section{References}

1. D'Angio GJ, Farber S, Maddock CL. Potentiation of x-ray effects by actinomycin D. Radiology. 1959;73:175-177.

2. Burris HA, Hurtig J. Radiation recall with anticancer agents. Oncologist. 2010;15(11):1227-1237.

3. Kodym E, Kalinska R, Ehringfeld C, et al. Frequency of radiation recall dermatitis in adult cancer patients. Onkologie. 2005;28(1):18-21.

4. Saif MW, Black G, Johnson M, et al. Radiation recall phenomenon secondary to capecitabine:possible role of thymidine phosphorylase. Cancer Chemother Pharmacol. 2006;58(6):771-775.

5. Yeo W, Johnson PJ. Radiation-recall skin disorders associated with the use of antineoplastic drugs. Pathogenesis, prevalence, and management. Am J Clin Dermatol. 2000;1(2):113-116.

6. Mizumoto M, Harada H, Asakura H, et al. Frequency and characteristics of docetaxel-induced radiation recall phenomenon. Int J Radiat Oncol Biol Phys. 2006;66(4):1187-1191.

7. Azria D, Magne N, Zouhair A, et al. Radiation recall:a well recognized but neglected phenomenon. Cancer Treat Rev. 2005;31(7):555-570.
8. Kitani H, Kosaka T, Fujihara T, et al. The "recall effect" in radiotherapy:is subeffective, reparable damage involved? Int J Radiat Oncol Biol Phys. 1990;18(3):689-695.

9. Camidge R, Price A. Characterizing the phenomenon of radiation recall dermatitis. Radiother Oncol. 2001;59(3):237-245.

10. Stein RS. Radiation-recall enteritis after actinomycin-D and adriamycin therapy. South Med J. 1978;71(8):960-961.

11. Schweitzer VG, Juillard GJ, Bajada CL, et al. Radiation recall dermatitis and pneumonitis in a patient treated with paclitaxel. Cancer. 1995;76(6):1069-1072.

12. Wallenborn PA, Postma DS. Radiation recall supraglottitis. A hazard in head and neck chemotherapy. Arch Otolaryngol. 1984;110(9):614-617.

13. Jeter MD, Janne PA, Brooks S, et al. Gemcitabine-induced radiation recall. Int J Radiat Oncol Biol Phys. 2002;53(2):394-400.

14. Maeng $\mathrm{CH}$, Park JS, Lee SA, et al. Radiation recall phenomenon presenting as myositis triggered by carboplatin plus paclitaxel and related literature review. J Cancer Res Ther. 2014;10:1093-1097.

15. Friedlander PA, Bansal R, Schwartz L, et al. Gemcitabine-related radiation recall preferentially involves internal tissue and organs. Cancer. 2004;100(9):1793-1799.

16. Corso CD, Ali AN, Diaz R. Radiation-induced tumor neoantigens:imaging and therapeutic implications. Am J Cancer Res. 2011;1(3):390-412.

17. Riaz N, Morris L, Havel JJ, et al. The role of neoantigens in response to immune checkpoint blockade. Int Immunol. 2016;28(8):411-419.

18. Eckardt MA, Bean A, Selch MT, et al. A child with gemcitabine-induced severe radiation recall myositis resulting in a compartment syndrome. $J$ Pediatr Hematol Oncol. 2013;35(2):156-161.

19. Squire S, Chan M, Feller E, et al. An unusual case of gemcitabine-induced radiation recall. Am J Clin Oncol. 2006;29(6):636.

20. Fakih MG. Gemcitabine-induced rectus abdominus radiation recall. JOP. 2006; 7:306-10

21. Barlesi F, Tummino C, Tasei AM, et al. Unsuccessful rechallenge with pemetrexed after a previous radiation recall dermatitis. Lung Cancer. 2006;54(3):423-425.

22. Lock M, Sinclair K, Welch S, et al. Radiation recall dermatitis due to gemcitabine does not suggest the need to discontinue chemotherapy. Oncol Lett. 2011;2(1):85-90.

23. Patel SC, Paulino AC, Johnston D, et al. Gemcitabine-induced radiation recall myositis in a patient with relapsed nasopharyngeal carcinoma. Pract Radiat Oncol. 2016;7(1):e19-e22. 